\title{
QUALITÄTSMANAGEMENT
}

\section{Orientierung für Praktiker}

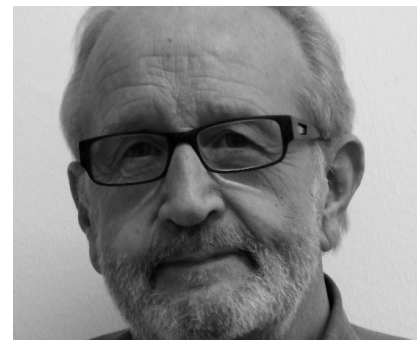

VON KARL H. WIESS Karl H. Wieß, gelernter Bankkaufmann, ist seit über zehn Jahren im sozialen Netzwerk in Frankfurt am Main in verschiedenen verantwortlichen Positionen tätig; zurzeit bei zwei Beschäftigungsinitiativen für Menschen mit Behinderungen.

E-Mail karl@wiess-frankfurt.de

\author{
Vor allem an kleinere und mittlere soziale Unterneh- \\ men richtet sich ein neues Werk zum Qualitätsmanage- \\ ment, die ein solches Vorhaben meist ohne viel externe \\ Hilfe beginnen und aufbauen müssen.
}

Der Ende letzten Jahres vorgelegte »Leitfaden für kleinere und mittlere Soziale Einrichtungen « beschreibt die Ansätze der DIN EN ISO 9000 ff., des EFQM-Modells und des KQS-Modells. Es zeigt bedeutsame Werkzeuge und Methoden des Qualitätsmanagements (QM) auf, erläutert den Zusammenhang von Qualitätsmanagement und Organisationsentwicklung, von Qualitätsmanagement und fachlicher Arbeit und von Qualitätsmanagement und Controlling.

Das Werk wendet sich also an Praktiker, aber auch an Studierende der Sozialen Arbeit und des Sozialmanagements in Bachelor- und Master-Studiengängen, die einen Einstieg in die Thematik erhalten und praxisbezogene Anwendungen und Umsetzungen kennenlernen wollen.

Die Autorin, Prof. Dr. Edeltraud Vomberg, unterrichtet an der Hochschule Niederrhein am Fachbereich Sozialwesen vor allem Qualitäts-, Projektund Kommunikationsmanagement. Weitere ihrer Lehr- und Forschungsgebiete sind Arbeitsmarktpolitik, Beschäftigungsförderung sowie Frauen als Unternehmensgründer und Frauen als Unternehmerinnen und in Führungspositionen.

Das Buch ist streng wissenschaftlich gegliedert. Es werden die diversen Modelle des Qualitätsmanagements analysiert und mit ihren unterschiedlichen Ansätzen und Grundsätzen unter Hinweis auf Beispielsliteratur dargestellt und miteinander verglichen. Dabei wird es aus meiner Sicht problematisch für interessierte Leserinnen und Leser, die die Fachsprache nicht beherrschen.

Unser Anliegen in sozialwirtschaftlichen Unternehmen ist es unter anderem, eine Zertifizierung im Qualitätsmanagement zu erhalten, um neben der Qualifikation der Mitarbeitenden die Berechtigung zur Teilnahme an öffentlichen Ausschreibungen zu erreichen. Dabei stehen wir stets vor der Problematik, wie die Qualität Sozialer Arbeit messbar zu machen ist.

\section{Sozialarbeit messen?}

Die Autorin bestätigt die Vermutung teilweise, dass exakte Messbarkeit im sozialen Bereich nicht möglich ist, da der Verdacht »in Teilen seine Berechtigung (hat), da sich Qualitätsmessung als komplexe Problematik darstellt «(S. 13). Gefolgt werden kann der Meinung der Autorin, dass »fachlich begründete und aus der Erfahrung wirksame Standards helfen, ein Minimum an Prozesssicherheit zu realisieren «(S. 15). Ebenso spielen »die aktive Einbindung und der ständige Dialog mit den Mitarbeitenden eine zentrale Rolle« (S. 17).

Die Darstellung des Sozialen Dreiecks aus Leistungserbringer, Leistungsempfänger und Kostenträger als auch andere Ausführungen erlauben die Anmerkung, dass nur - allerdings wesentliche - Teile des Buches für das Qualitätsmanagement bei einigen Einrichtungen, beispielsweise bei anerkannten Integrationsunternehmen, von Bedeutung sind. Denn, so die Aussage der Autorin: »Soziale Dienstleistungs- 
organisationen bedienen mit dem Aufbau von Controlling einerseits sowohl den Legimitationsdruck, unter dem sie öffentlich stehen, als auch den zunehmenden Wettbewerbsdruck, ihre Angebote an den Kunden (Kostenträger oder Klienten) bringen zu müssen, um am Markt zu bestehen ...«(S. 265). Integrationsunternehmen für Menschen mit Behinderungen müssen im Rahmen eines Vierecks geführt werden: Ich würde diese Komponente als » Marktpreis der Produkte der betreuten Mitarbeitenden « bezeichnen wollen.

Ermutigend ist der Hinweis zu lesen: »Es spielt keine Rolle, ob diese Instrumente und Methoden mit oder ohne ein Rahmenmodell des QM eingesetzt werden. «(S. 141) Und somit schließe ich mich gern dem Schlusswort der Verfasserin an: "Aber genau das macht Spaß am QM. Es gibt immer wieder etwas Neues zu tun und zu gestalten. Und es gibt immer wieder Überraschungen. «

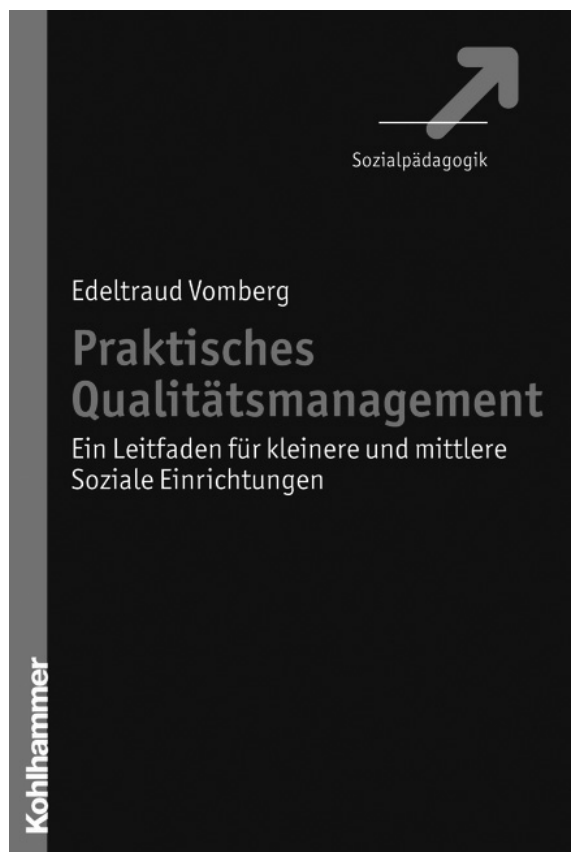

Edeltraud Vomberg: Praktisches Qualitätsmanagement. Ein Leitfaden für kleinere und mittlere Soziale Einrichtungen. Kohlhammer Verlag, Stuttgart 2010. 300 Seiten. 29,80 Euro. ISBN 978-3-17-020619-9.

\section{Zum 70. Geburtstag von Bernd Maelicke}

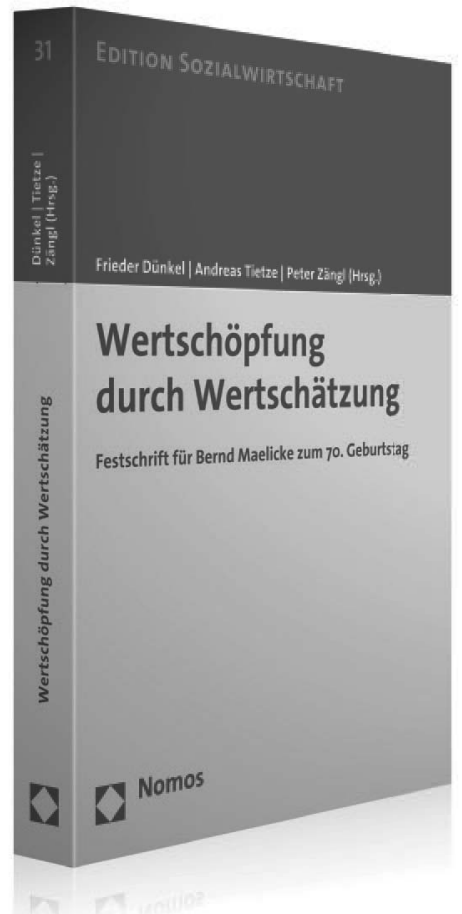

\section{Wertschöpfung durch Wertschätzung}

Festschrift für Bernd Maelicke zum 70. Geburtstag

Herausgegeben von Prof. Dr. Frieder Dünkel, Dr. Andreas

Tietze und Prof. Dr. Peter Zängl 2011, 191 S., brosch., 29,- $€$ ISBN 978-3-8329-6534-1

(Edition Sozialwirtschaft, Bd. 31)

Diese Festschrift erscheint zum 70. Geburtstag von Bernd Maelicke, dem Initiator und ersten Herausgeber der „Edition Sozialwirtschaft“ im Nomos Verlag.

Der Titel „Wertschöpfung durch Wertschätzung“ war nicht nur das Thema seiner Antrittsvorlesung an der Leuphana Universität Lüneburg - er verweist zugleich auf ein Markenzeichen seines beruflichen und privaten Wirkens: der Optimierung und Vernetzung von fachlichen und persönlichen Potentialen zu gemeinsamen Handlungsstrategien mit dem Ziel der Förderung von sozialer Gerechtigkeit.

Die Grundlagenbeiträge und die Erinnerungen von Weggefährten machen deutlich, in welcher Breite und Tiefe Bernd Maelicke Soziale Innovationen erreichen bzw. fördern konnte - unter je spezifischen gesellschaftlichen und institutionellen Rahmenbedingungen. So entsteht nicht nur eine Zeitgeschichte der alten und neuen Bundesrepublik, exemplarisch wird an einer Nachkriegsbiographie deutlich, welche nachhaltigen Wirkungen der „Marsch durch die Institutionen“ bewirken konnte.

Für Fach- und Führungskräfte in Sozialen Organisationen legen die Herausgeber hiermit ein sowohl grundlegendes wie spannendes „Lesebuch“ vor, dass anregen und ermuntern will, sich weiterhin für Soziale Innovationen zu engagieren und die dafür notwendigen strategischen und operativen Prozesse hochprofessionell nach dem Konzept von Forschung und Entwicklung zu steuern.

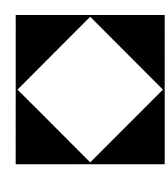

Nomos

Bitte bestellen Sie im Buchhandel oder versandkostenfrei unter $>$ www.nomos-shop.de 